\title{
Performative Religionspädagogik im Horizont kunstdidaktischer Performancearbeit
}

\author{
Claudia Gärtner
}

Die jahrzehntelange gepflegte Aufgabenteilung zwischen Religionsunterricht (RU) und Katechese ist in vielerlei Hinsicht obsolet geworden. Wenn man früher davon ausging, dass Kinder in der Kirchengemeinde oder der Familie in den Glauben eingeführt wurden, dann konnte sich der Beitrag des RU primär darauf konzentrieren, die dort erlangten Kenntnisse und Erfahrungen zu reflektieren. Zwar war diese Aufteilung aus theologischer wie auch rein praktischer Perspektive niemals unproblematisch, aber mit dem mittlerweile unübersehbar gewordenen Traditionsabbruch treten die Schwierigkeiten deutlich zu Tage. Was bleibt einem RU, wenn keine vorgängigen religiös relevanten Erfahrungen und Kenntnisse von der (christlichen) Religion vorhanden sind? Dem radikalen Abbruch christlicher Traditionen versuchen Religionspädagoginnen und -pädagogen auf unterschiedlichen Wegen zu begegnen. In den letzten Jahren tritt hierbei insbesondere die so genannte „Performative Religionspädagogik“ hervor, die Religion im Umgang mit religiösen Vollzügen, in der raum-leiblichen Übernahme religiöser Perspektiven für die zu bildenden Subjekte erfahrbar und begreifbar machen will. In diesem Sinne bringt sie die Formen der gelebten Religion im RU „performativ“ zur Darstellung. Der Begriff „performativ“ ist dabei jedoch in der Religionspädagogik „hochgradig äquivok. “1 Auch in den Kulturwissenschaften, die schon seit einigen Jahren einen „performative turn“ vollzogen haben, scheint der Begriff vielmehr ein „umbrella term “2 zu sein, unter dem sich viel Unterschiedliches versammeln lässt.

1 Rudolf Englert, Performativer Religionsunterricht - eine Zwischenbilanz, in: Zeitschrift für Pädagogik und Theologie 60, 2008, 3-16, 9. Lämmermann spricht in dieser Hinsicht von erheblichen, wenn nicht sogar unaufhebbaren Unterschieden. Vgl. Godwin Lämmermann, Reli auf der Show-Bühne. Eine polemische Kritik der sich „performative“ nennenden „Religionsdidaktik“, in: Theo-web. Zeitschrift für Religionspädagogik 7, 2/2008, 107-123, 113.

2 Vgl. Uwe Wirth, Der Performanzbegriff im Spannungsfeld von Illokution, Iteration und Indexialität, in: ders. (Hg.), Performanz. Zwischen Sprachphilosophie und Kulturwissenschaft, Frankfurt a.M. (Suhrkamp) 2002, 9-60, 10; Erika Fischer-Lichte, Ästhetik des Performativen, Frankfurt a.M. (Suhrkamp) 2004, 41. 
„Performanz kann sich ebenso auf das ernsthafte Ausführen von Sprechakten, das inszenierende Auffübren von theatralen oder rituellen Handlungen, das materiale Verkörpern von Botschaften im ,Akt des Schreibens' oder auf die Konstitution von Imaginationen im ,Akt des Lesens' beziehen. “3

Dieses unterschiedliche Begriffsverständnis ist nicht nur im wissenschaftstheoretischen Diskurs zu konstatieren, sondern führt in der Religionspädagogik zu höchst unterschiedlichen didaktisch-hermeneutischen Ausprägungen - mit je unterschiedlichen Stärken und Problemstellungen. Im Folgenden möchte ich daher verschiedene Interpretationen des Performanzbegriffs innerhalb der performativen Religionspädagogik erörtern. Dabei wird deutlich, dass unterschiedliche sprach-, kultur- und theaterwissenschaftliche Lesarten in den jeweiligen religionspädagogischen Ansätzen ihren Niederschlag gefunden haben.

Erstaunlicher Weise bleiben in den religionspädagogischen Debatten kunstwissenschaftliche und kunstdidaktische Überlegungen weitgehend unberücksichtigt, obwohl die ästhetische Dimension performativen Lernens dort stets betont wird. Zudem stellt Performance sowohl ein bedeutendes künstlerisches als auch kunstpädagogisches Prinzip dar. Es ist daher lohnenswert, einen Blick in die künstlerische und didaktische Performancearbeit zu werfen. Dadurch strebe ich weniger eine zusätzliche Begriffserweiterung an, sondern vielmehr eine Schärfung der performativen Religionspädagogik - auch in Hinblick auf entsprechende Problemstellungen.

In einem ersten Schritt skizziere ich verschiedene Spielarten der performativen Religionspädagogik, ohne hierbei jedoch auf Vollständigkeit zu zielen oder eine weitere Zwischenbilanz zu den entsprechenden Debatten geben zu wollen. Sondern es geht mir primär darum, den Zusammenhang von unterschiedlichem Begriffsverständnis und didaktisch-hermeneutischer Ausformulierung zu erörtern. In einem zweiten Schritt stelle ich wesentliche Aspekte eines künstlerischen Performancebegriffs und entsprechende Überlegungen zu einer performanceorientierten Kunstdidaktik dar. Abschließend blicke ich im Lichte dieses Performanceverständnisses erneut auf strittige Aspekte der Performativen Religionspädagogik.

\section{Religionspädagogik unter dem „umbrella“ des Performativen}

Die Bezeichnung „performative Religionspädagogik“ verweist forschungsgeschichtlich zuerst auf den von John L. Austin geprägten Neologismus „performativ“, auch wenn einige Vertreterinnen und Vertreter performativer Religionspädagogik Austins Begriffsverständnis als zu enggeführt ab-

3 Wirth (Anm. 2), 9. 
lehnen. ${ }^{4}$ Austin prägt diesen Begriff im Rahmen seiner Sprechakttheorie, die gerade im theologischen Diskurs von Gewicht ist. Er leitet „performativ" von „to perform “ im Sinne von „man, vollzieht" Handlungen“" ab und macht damit darauf aufmerksam, dass Sprechen auch Handlung ist. Performative Sprechakte sind nach Austin wirklichkeitskonstituierende und selbstreferenzielle sprachliche Vollzüge (wie z.B. Segen oder Taufe), wenn diese in Ernsthaftigkeit und Aufrichtigkeit vollzogen werden. Obwohl er später die Unterscheidung von performativem und konstativem Sprechen zugunsten der Dreiteilung in lokutionäre, illokutionäre und perlokutionäre Sprechakte auflöst, ${ }^{6}$ so lässt er hiermit noch nicht die „Definition fragwürdig werden, die er unter Bezug auf die ursprüngliche Performative vom Begriff des Performativen gegeben hat: nämlich daß dieser (Sprech-)Handlungen meint, die selbstreferentiell und wirklichkeitskonstituierend sind "7. Im Rahmen der Performativen Religionspädagogik scheinen neuerdings besonders katholische Vertreterinnen und Vertreter an diesem Verständnis von Performativität anzuknüpfen. In Anlehnung an Austin ist hierbei die Annahme grundlegend, dass sich im Sprechen von religiösen Texten, im Vollzug religiöser Handlungen oder im In-Gebrauch-Nehmen von religiösen Zeichen Religion vollzieht bzw. wahrnehmbar wird. In dieser Hinsicht sollen SchülerInnen aus der Teilnehmerperspektive, die zumindest für die Lehrkräfte vorausgesetzt wird, mit Vollzugsformen gelebten Glaubens vertraut gemacht werden. ${ }^{8}$ Dieses Vertrautmachen zielt letztlich auf mystagogische Einführung in den Glauben und auf kirchliche Partizipation. „Mystagogie kann ganz einfach sein. Wir lehren die Kinder, wie Weihwasser zu nehmen sei, wie eine Kirche zu betreten sei, wie und warum eine Kniebeuge zu machen sei [...]. Wir lehren sie, mit Orten und Zeiten der Stille umzugehen, zu gehen, zu stehen, zu beten und zu singen. Wir verhelfen

4 Vgl. Bernhard Dressler/Thomas Klie, Strittige Performanz. Zur Diskussion um den performativen Religionsunterricht, in: Thomas Klie/Silke Leonhard, Performative Religionsdidaktik. Religionsästhetik - Lernorte - Unterrichtspraxis, Stuttgart (Kohlhammer) 2008, 210-224, 218 (Erstveröffentlichung in: Pastoraltheologie 96, 2007, 241-254). Vgl. zum Zusammenhang von Performativem RU und John L. Austin Hanna Roose, Performativer Religionsunterricht zwischen Performance und Performativität, in: Loccumer Pelikan, 2006, 110-115.

5 Vgl. John L. Austin, Zur Theorie der Sprechakte (How to do things with Words), Stuttgart (Reclam) 1979, 30.

6 Vgl. ebd., $112 \mathrm{ff}$.

7 Fischer-Lichte (Anm. 2), 33. Der Begriff des Performativen geht bei Austin nun vorrangig in den Begriff des illokutionären Akts ein. Explizite Performativa qua institutionelle Sprechakte (so z. B. Segen, Eheschluss usw.) bilden dann nur noch eine Sonderklasse der Illokution (vgl. Austin (Anm. 5), 164 f.)

8 Vgl. Deutsche Bischofskonferenz (Hg.), Der Religionsunterricht vor neuen Herausforderungen, Bonn 2005, $23 \mathrm{f}$. 
ihnen zum Erlebnis einer singenden und betenden Gemeinschaft. “9 Ein so verstandenes performatives Lernen will „bei den Schüler/innen einen Suchprozess auszulösen, damit eine Performation, ein ,Durch-Bilden` auf Gott hin einen Anfang nehmen kann “10. Hierdurch sollen Möglichkeiten für die Begegnung mit dem lebendigen Gott eröffnet werden. Doch wie wiederum vollzieht sich dieses Einbringen von religiösen Texten, Ritualen oder Zeichen in (schulische) Lernprozesse? Handelt es sich hierbei primär um den Nach- und Mitvollzug „authentischer" Formen von Religion, also mit Austin gesprochen um ernsthafte und aufrichtige Handlungen? Dann stellt sich insbesondere im Rahmen des schulischen Religionsunterrichts die Frage, ob hier die Schülerinnen und Schüler nicht missionarisch vereinnahmt werden. Oder verstehen die Religionslehrerinnen und -lehrer bzw. die Schülerinnen und Schüler dies primär als ein didaktisch inszeniertes, spielerisches Probehandeln? Dann wäre dies ein - im Sinne Austins - unernster und damit nichtiger Gebrauch religiöser Sprache und Zeichen. Der Religionsunterricht liefe damit Gefahr, die Vollzugsformen zu profanisieren oder zu musealisieren. ${ }^{11}$ Die Kunst eines so ausgerichteten performativen Religionsunterrichts scheint es zu sein, den Unterricht zwischen den Klippen von „Missionierung“ und „Profanisierung“ zu navigieren. Um dies zu erreichen, wird ein besonderer Fokus auf die Reflexion einer performativen Praxis gelegt, die begleitend erfolgen und im Verlauf der Schullaufbahn einen immer größeren Anteil einnehmen soll. ${ }^{12}$

Eine andere Ausprägung performativer Religionspädagogik setzt an einem kulturwissenschaftlichen Performanceverständnis (z.B. von Victor Turner) an, das explizit auf einer spielerischen - und damit nach Austin unernsten - Inszenierung fremder Rituale beruht. Im Rahmen ethnografischen Lernens geht man etwa davon aus, dass durch eine Performance, durch eine dramatische Aufführung von fremden Ritualen, Erfahrungen gemacht wer-

9 Eckhard Nordhofen, Diskursive und performative Mystagogie. Über das, was im Religionsunterricht gesagt, und das was nicht gesagt werden kann, in: Informationen für Religionslehrerinnen und Religionslehrer 36, 2007, 7-14, 14.

10 Mirjam Schambeck, Religion zeigen und Glauben lernen in der Schule? Zu den Chancen und Grenzen eines performativen Religionsunterrichts, in: Religionspädagogische Beiträge 58, 2007, 61-80, 72. Dabei ist sich Schambeck der Grenzen eines solchen Unterfangens im Rahmen des schulischen RUs durchaus bewusst. Vgl. ebd., $75 \mathrm{ff}$.

11 Vgl. Roose (Anm. 4), 112. Vgl. a. Englert (Anm. 1), 9.

12 Vgl. Nordhofen (Anm. 9), 14. Mendl verweist angesichts dieser Problemstellung auf Austins Unterscheidung von lokutionären, illokutionären und perlokutionären Sprechakten. Denn hierdurch würde deutlich, dass eine gemeinsam vollzogene performative Handlung individuell gedeutet wird. So liege es „in der Entscheidung des Einzelnen, das gemeinsame Sprechen eines Gebets (als lokutionärer Akt) auch mit der Überzeugung zu verbinden, dass damit der beschriebene illokutionäre Akt ,Beten' vollzogen wird." Hans Mendl, Religion erleben. Ein Arbeitsbuch für den Religionsunterricht. 20 Praxisfelder, München (Kösel) 2008. Der perlokutionäre Effekt hänge nicht von der Absicht des Sprechers oder des Inhalts der Äußerung ab. 
den, die in der nachgängigen Reflexion zum Verstehen der Fremdkultur beitragen. Dabei plädiert Turner für einen Dreischritt: „die Umwandlung der Ethnographie in ein Bühnenstück, des Stücks in eine Aufführung, der Aufführung in eine Meta-Ethnographie “13. Hierbei sollen fremde kulturelle Bräuche und Rituale nicht mimetisch nachgespielt, sondern poietisch „von innen“ wiedererschaffen werden. ${ }^{14}$ Dies gelingt nach Turner am ehesten durch eine dialektische Beziehung von Lernen über und Darstellung von einer fremden Kultur. Diese dialektische Bewegung führe zu einer komplexen Aufführung (Performance), in der sich die Darstellenden im Fluss („Flow“) befänden und existenzielle Erfahrungen machten, die in der Reflexion sowohl zu einem vertieften Verständnis unseres Selbst als auch fremder Kulturen führten. ${ }^{15}$

Harald Schroeter-Wittke knüpft mit seiner Lesart einer performativ ausgerichteten Religionsdidaktik an einen solchen kulturwissenschaftlich geprägten Performancebegriff an. Er zeigt auf, dass der performative Grundgedanke mit der „Schleiermacher'schen Einsicht Ernst macht, dass es in der Religion keine Mitteilung ohne Darstellung und auch keine Darstellung ohne Mitteilung gibt. “"16 Nur im darstellenden Handeln kann sich demnach Religion wirksam mitteilen. Für die religiöse Rede stellt Schleiermacher daher die Forderung auf: „Wir verlangen eine Wirkung die etwas actives ist und den Impuls der im darstellenden ist fortpflanzt auf die für die er darstellt. “ ${ }^{17}$ Nach Schroeter-Wittke liegen gerade hierin die Parallelen zur Performance, denn in der Performance wird deutlich, dass die Grenzen zwischen Darstellung und Wirksamkeit, zwischen Bühne und Publikum aufgelöst werden. ${ }^{18}$ Es lässt sich also im gelingenden Fall nicht länger zwischen Inhalt und Form, zwischen handelndem Subjekt und Handlung unterscheiden. In der Performance entsteht vielmehr erst die Religion der daran beteiligten Subjekte und kommt darin zur Darstellung. ${ }^{19}$ Bedeutsam ist für Schroeter-Wittke dabei die Verknüpfung von Handlung und Reflexion,

13 Victor Turner, Dramatisches Ritual, rituelles Theater. Performative und reflexive Ethnologie, in: Wirth (Anm. 2), 193-209, 194.

14 Vgl. ebd., 198.

15 Vgl. ebd., $208 \mathrm{f}$.

16 Harald Schroeter-Wittke, Mission als Ver-Fahren. Praktisch-theologische Anmerkungen zu einer performativen Religionspädagogik, in: Michael Böhme u.a. (Hg.), Mission als Dialog. Zur Kommunikation des Evangeliums heute, Leipzig (Evangelische Verlagsanstalt) 2003, 151-168, 165.

17 Friedrich Schleiermacher, Die praktische Theologie nach den Grundsätzen der evangelischen Kirche im Zusammenhange dargestellt, hg. v. Frerichs, J., Berlin 1850, 37, hier zit. n. Harald Schroeter-Wittke, Praktische Theologie als Performance. Ein religionspädagogisches Programmheft mit 7 Programmpunkten, in: Eberhard Hauschildt/Ulrich Schwab (Hg.), Praktische Theologie für das 21. Jahrhundert, Stuttgart (Kohlhammer) 2002, 143-159, $145 \mathrm{f}$.

18 Vgl. Schroeter-Wittke (Anm. 17), $145 \mathrm{f}$.

19 Vgl. Schroeter-Wittke (Anm. 16), 62. 
die in dem kulturwissenschaftlichen Verständnis von Performance zusammenkommen. ${ }^{20}$ Darüber hinaus erweist sich für ihn der in der Performance immer wieder neu entstehende Bedeutungsüberschuss als zentral. Denn dieser ist weder im Vorfeld didaktisch kalkulierbar noch im Nachhinein objektivierbar, sondern er entsteht vielmehr in der Performance stets neu. Daher lassen sich Performances zwar wiederholen, ohne dass sie aber zum selben Ergebnis führen ${ }^{21}$ In dieser Hinsicht gehen Schroeter-Wittke viele Ansätze der Performativen Religionspädagogik nicht weit genug, denn diese „fassen [...] die Brisanz der Tatsache, dass die Performance die Religion der daran beteiligten Subjekte allererst entstehen und zur Darstellung kommen lässt, zumeist nicht gebührend ins Auge. “22 Vielmehr hielten sie an dem Charakter der Aufführung, der Inszenierung (von der Lehrperson) vorgegebener Inhalte fest.

Auch wenn man davon ausgeht, dass Religion im performativen Vollzug erst entsteht und zur Darstellung kommt, dann ist dennoch fraglich, ob aus der performativen Grundstruktur von Religion ein didaktisches Prinzip abgeleitet werden kann. Erhellend mag dabei erneut ein Blick auf die von Turner geschilderte ethnografische Performancearbeit sein. ${ }^{23}$ Ausgehend von der Erkenntnis, dass Rituale für das Verständnis von fremden Kulturen unverzichtbar sind, werden diese - unter Einbeziehung von Wissen und Kenntnissen über die Kultur - inszeniert und anschließend ausgewertet. Turners Schilderungen verdeutlichen, dass die Teilnehmerinnen und Teilnehmer tief greifende Erfahrungen und neue Einsichten über Ritual und Kultur erlangt haben. Dennoch ist offensichtlich, wie unhintergehbar der persönliche Horizont der Darstellerinnen und Darsteller bei einer solchen Performance ist. Inwiefern die hierbei gemachten Erfahrungen qualitativ auf die Ursprungskultur der dargestellten Rituale verweisen, bleibt offen. Zwar ist den SchülerInnen die christliche Kultur und Religion in der Regel nicht so fremd wie die in den ethnografischen Beispielen betrachteten Kulturen. Aber gleichwohl stellt sich die Frage, welche Erfahrungen die Schülerinnen und Schüler in didaktisch inszenierten Performances im RU machen können. Auch wenn Erfahrungen didaktisch nie kalkulierbar sind, so muss in einem solchen Lernsetting reflektiert werden, dass didak-

20 Vgl. Harald Schroeter-Wittke, Performance als religionsdidaktische Kategorie. Prospekt einer performativen Religionspädagogik, in: Thomas Klie/Silke Leonhard (Hg.), Schauplatz Religion. Grundzüge einer Performativen Religionspädagogik, Leipzig (Evangelische Verlagsanstalt) 2002, 47-66, 48 mit Bezug auf Christoph Wulf/Jörg Zirfas, Sprache, Macht und Handeln, in: dies (Hg.), Grundlagen des Performativen. Eine Einführung in die Zusammenhänge von Sprache, Macht und Handeln, Weinheim/München (Juventa) 2001, 9-24.

21 Vgl. Schroeter-Wittke (Anm. 20), 49; 58.

22 Ebd., 62. „Dies genau bezeichnet die Problematik, die viele kirchliche Theologien mit dem performative turn haben, weil hier nämlich die Erkenntnis dämmert, dass Bedeutungen durch Performances allererst entstehen.“ (Ebd., 51.)

23 Vgl. Anm. 13. 
tisch inszenierte Performances ggf. zwar bildungsrelevante Erfahrungen ermöglichen, aber religiös entkontextualisiert eventuell zu Erlebnissen werden, die nur schwerlich zu einer Sensibilisierung für Religion oder religiöse Erfahrungen beitragen.

Da im Bereich religionspädagogischer Performancearbeit bislang wenige theoretische Überlegungen und praktische Erfahrungen mit Performance vorliegen, werden diese Anfragen im Horizont kunstdidaktischer Überlegungen im dritten Teil erneut aufgegriffen.

Eine dritte Lesart der Performativen Religionspädagogik nimmt vornehmlich eine theaterwissenschaftliche Perspektive ein. Dabei soll die Performative Religionspädagogik weder auf Austins Verständnis von „Performativität" eingeengt, ${ }^{24}$ noch explizit die Offenheit eines kulturwissenschaftlichen Performancebegriffs angezielt werden. Vielmehr ist hierbei die Performative Religionspädagogik in Hinblick auf ein allgemeines Verständnis von Performanz ausgerichtet. „,Performanz kann nun stehen für die willentliche Ausführung eines Sprechaktes, für die Inszenierung theatraler oder ritueller Handlungen sowie für die Verkörperung imaginierter Bot-

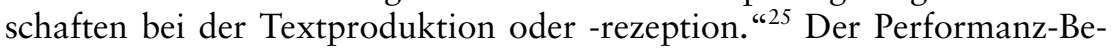
griff steht hier für „einen Vorgang, bei dem sich etwas als Äußerung verkörpert" ${ }^{26}$ und meint allgemein ein leib-räumliches Vorzeigen, wie z. B. vorlesen, vorspielen oder vormalen. Diese Vorgänge werden als elementare leibräumliche Deutungsakte verstanden ${ }^{27}$, damit stehe „performativ“ für vielfältige unterrichtliche Zeige-Handlungen. ${ }^{28}$ Mit diesem sehr weiten Performanzbegriff positionieren sich Klie und Dressler zwischen bzw. jenseits der aufgewiesenen Pole von Performativität im Sinne Austins und Performance. Da sie davon ausgehen, dass Religion das ist, „was sich in, mit und unter den Formen ihrer Ingebrauchnahme zeigt [...], was sich performiert, in Erscheinung tritt, ${ }^{\text {" } 29}$ müssen nach ihrer Ansicht in didaktischen Prozessen

24 In diesem Sinne grenzen sich Thomas Klie und Bernhard Dressler von Hanna Rooses Verweis auf Austins Sprechakttheorie ab. Mit ihrem Fokus auf den Performativitätsbegriff von Austin übersehe Roose nach Ansicht von Dressler und Klie die Semiotizität des Performanzbegriffs (vgl. Dressler/Klie (Anm. 4), 218). Mit Cassirer gehen sie von der Unterscheidung in Darstellungsform und Ausdrucksform aus. Die Ausdrucksform trennt demnach Zeichen und bezeichnete Sache noch nicht strikt, so dass ein Zeichen zugleich noch das ist, worauf es verweist. Die Darstellungsform hingegen stelle eine referentielle Beziehung zwischen Zeichen und Bezeichnetem dar. Austin bezöge sich mit seinem Performativitätsbegriff auf die Darstellungs- und nicht die Ausdrucksform, Performanz beziehe sich aber auf die Ausdrucksform. (Vgl. ebd., 217 f)

25 Thomas Klie/Bernhard Dressler, Performative Religionspädagogik - Rezeption und Diskussion 2002-2008, in: Klie/Leonhard (Anm. 4), 225-236, 226.

26 Ebd., 226.

27 Vgl. Dressler/Klie (Anm. 4), 218.

28 Vgl. Klie/Dressler (Anm. 25), 225.

29 Silke Leonhard/Thomas Klie, Ästhetik - Bildung - Performanz. Grundlinien performativer Religionsdidaktik, in: dies. (Anm. 4), 9-25, 11. 
eben diese Formen inszeniert und zur Probe erschlossen werden. Inszenierung wird zum grundlegenden didaktisch-hermeneutischen Prinzip und Verfahren. Religiöse Lernarrangements besitzen in diesem Verständnis eine grundsätzliche Strukturanalogie zum Theater, ${ }^{30}$ gelernt wird, wenn bzw. was inszeniert und gezeigt wird. „Es liegt sachlich nahe, sich hierbei an der Theater-Didaktik zu orientieren, an den Bedingungen der Möglichkeit, theatrale Texte zu inszenieren, genauer: zu re-inszenieren. “31

In dieser Perspektive stellen sich die oben aufgezeigten Fragen von Missionierung und Profanisierung nicht. Denn entsprechend des zu Grunde gelegten semiotischen Religionsverständnisses gibt es jenseits der Ingebrauchnahme von Formen der konkreten Religion keine „substantielle“ Religion, die sich profanisieren oder musealisieren ließe. Zeichen oder Formen „werden religiös im Gebrauch. “ ${ }^{32}$ Bleibt man in der Logik dieses Religionsverständnisses, das durchaus hinterfragt werden könnte, so stellen sich dennoch Anfragen. Wie müssen Zeichen und Formen einer Religion inszeniert (und reflektiert) werden, dass man auch ohne religiöse Vorerfahrungen oder zumindest ohne eine religiöse Offenheit überhaupt eine Ahnung von ihrem religiösen Gebrauch erhält? Wie viel Vorerfahrungen und Vorkenntnisse muss man besitzen, um religiöse Zeichen sachangemessen inszenieren bzw. In-Gebrauch nehmen zu können ?33

Ausschlaggebend erscheint damit weniger, dass religiöse Formen gezeigt oder inszeniert werden, ,sondern wie die Inszenierung des Experimentierfeldes ,Religionsunterricht ${ }^{\star}$ zustande kommt. “34 In dieser Hinsicht führen m. E. theaterwissenschaftliche Differenzierungen im Inszenierungsbegriff weiter. Nach Erika Fischer-Lichte erfüllt das Theater immer zugleich eine referentielle und eine performative Funktion. „Während die referentielle Funktion auf die Darstellung von Figuren, Handlungen, Beziehungen, Situationen etc. bezogen ist, richtet sich die performative auf den Vollzug von Handlungen - durch die Akteure und zum Teil auch durch die $\mathrm{Zu}-$ schauer - sowie auf ihre unmittelbare Wirkung. “" ${ }^{35}$ Dabei treten im Verlauf

30 Vgl. Klie/Dressler (Anm. 25), 229.

31 Dressler/Klie (Anm. 4), 215.

32 Bernhard Dressler, Religion und Bildung in den Differenzen des Lebens. Zeitschrift für Pädagogik und Theologie 59, 2007, 269-286, 280.

33 Vgl. Hanna Roose, Religiöse Praxis in der Grundschule. Eine Standortbestimmung im Rahmen der Debatte zum performativen Ansatz, in: Loccumer Pelikan 2008, 103-110, 107.

34 Ursula Roth, Von der Inszenierung bis zur Performativität. Der Religionsunterricht im Lichte kulturwissenschaftlicher Grundkategorien, in: Klie/Leonhard (Anm. 4), 38-50, 40.

35 Erika Fischer-Lichte, Grenzgänge und Tauschhandel. Auf dem Wege zu einer performativen Kultur, in: Wirth (Hg.) (Anm. 2), 277-300, 279. Nach Uwe Wirth bleibt hier jedoch kritisch zu hinterfragen, „ob die Differenzierung zwischen der ,performativen“ und der ,referentiellen` Funktion nicht hinter Austin zurückfällt, insofern sie seine Revision der Unterscheidung performativ/konstativ nicht berücksichtigt.“ (Wirth (Anm. 2), 39.) 
der Theatergeschichte diese beiden Funktionen unterschiedlich deutlich zu Tage. Bis zur Mitte des vergangenen Jahrhunderts dominierte nach FischerLichte die referentielle Funktion, die (werktreue) Darstellung von Personen und Stücken stand im Vordergrund. In den letzten Jahrzehnten erhielten hingegen die je neu vollzogene Darstellung eines Stücks, die leibliche KoPräsenz und Aktivität von Darsteller und Zuschauer, die Prozesshaftigkeit der Wahrnehmung und Darstellung mehr Gewicht. Performativ ausgerichteten Inszenierungen sei dabei ein spezifischer Realitätsakzent zu Eigen, sie konstituierten Wirklichkeit. ${ }^{36}$ In dieser Perspektive bestünde daher auch kein grundlegender Unterschied zwischen Theaterinszenierung und Performance, da Theater immer auch performative Elemente besäße, ohne jedoch mit Performance deckungsgleich zu sein. ${ }^{37}$ Folgt man Fischer-Lichtes Unterscheidung von referentiellen und performativen Dimensionen von Theaterinszenierungen, dann lassen sich die unterschiedlichen Spielarten Performativer Religionspädagogik graduell unterscheiden. In dieser Lesart betont z.B. Schroeter-Wittke insbesondere die performative Vollzugsdimension, während andere Ansätze stärker die referentielle Dimension und die Inszenierung bestimmter religiöser Formen oder Texte im Blick haben.

Wenn also Inszenierung als ein Grundprinzip und didaktisch-methodisches Verfahren von Religionspädagogik betrachtet wird, dann stellt sich die Frage, wie deutlich die referentielle bzw. die performative Dimension betont werden. Die referentielle Dimension kommt primär im Nachspielen von religiösen Texten, in der Verwendung von vorgegebenen religiösen Formen und Zeichen zum Tragen. Religion wird gezeigt, indem sie nachgespielt wird. Konkretisiert man diese Unterscheidungen in Hinblick auf Inszenierungen im RU, so müssten analog zu Theaterinszenierungen die verschiedenen Dimensionen und Faktoren jeweils neu zueinander in Beziehung gesetzt werden. So stellen sich z. B. Fragen nach dem Verhältnis von Textbezug (referentielle Funktion) und Darstellung (performative Funktion). Lotet die Lehrperson dieses Verhältnis (als „Regisseur“) aus? Wie viel Einfluss nimmt sie auf die Inszenierung, wie ist die Zusammenarbeit mit den Darstellerinnen und Darstellern? Stehen Darstellung und Handlungsvollzug oder das Nachspielen des Stücks im Vordergrund?

In dieser Perspektive ließe sich aus theaterwissenschaftlicher Sicht auch die im Kontext der Performativen Religionspädagogik geäußerte Gefahr einer durch Rollenübernahme und Inszenierung bewirkte Vereinnahmung oder Missionierung der Schülerinnen und Schüler neu beleuchten. Denn hier stellt sich - wenn auch deutlich abgeschwächt - die Frage, inwiefern sich Schauspieler mit ihren Rollen identifizieren. Ein Blick auf die Geschichte der Schauspielkunst kann hier weiterführen. Dort reicht z. B. das Verhältnis von Schauspielerin und Rolle von der totalen Identifikation

36 Vgl. Roth (Anm. 34), 48 f.

37 Vgl. Fischer-Lichte (Anm. 35), 296. 
bis hin zu bewusst verwendeten Verfremdungs- und Brechungsstrategien (z.B. bei Brecht). ${ }^{38}$ Um Vereinnahmung und vorschnelle Identifikation mit religiösen Formen zu vermeiden und eine reflexive Distanz zu ermöglichen, „bietet es sich an, sich auch im Religionsunterricht Verfremdungstechniken wie etwa derjenigen Bertolt Brechts zu bedienen: verfremdende, unkonventionelle Darstellungsformen auszuprobieren, die szenische Darstellung immer wieder durch Pausen zur reflexiven Betrachtung zu unterbrechen etc. “ 39

Darüber hinaus verdeutlichen die Strukturanalogien zum Theater, dass in der Performativen Religionspädagogik die Bedeutung der Zuschauer kaum reflektiert wird ${ }^{40}$ obwohl das Publikum eine ebenso unverzichtbare Position innehat wie die Schauspielerinnen und Schauspieler. In diesem Sinne ist Zuschauen selbst eine Form aktiven Handelns, „Rezipieren ist Produzieren, Zuschauen ist Handeln " ${ }^{\text {41 }}$. Analog wäre nach der jeweiligen Bedeutung der Zuschauer im Performativen Religionsunterricht zu fragen.

Die performative Dimension erhält nach Ursula Roth aus theaterdidaktischer Perspektive dann ein besonderes Gewicht, wenn Unterrichtsprozesse so inszeniert werden, „dass das Wechselspiel von Zeichenproduktion und Zeichenrezeption alle Beteiligten in ihren leibhaften Erfahrungen zusammenbindet und Rückkopplungen wie auch der Rollenwechsel zwischen Akteuren und Publikum provoziert würden. Didaktische und methodische Entscheidungen wären so zu treffen, dass der transitorische Aspekt der Performanz zum Tragen käme [...]. Schließlich müsste vor allem der transformative Aspekt der Performativität zur Geltung kommen, indem Erfahrungsräume hergestellt und eingerichtet würden, in denen Schüler und Schülerinnen, aber auch Lehrerinnen und Lehrer solche Erfahrungen machen können, die eingespielte Verstehensmuster von Wirklichkeit aufweichen und $[. .$.$] durch andere, teilweise quer dazu stehende Deutungsmuster$ überlagert werden." ${ }^{42}$

Diese Anforderungen an einen performativ ausgerichteten RU lassen sich m. E. mit Blick auf die Kunstdidaktik näher erörtern. Hierdurch können sowohl die von Roth eingeforderten Aspekte performativen Unterrichts konkretisiert, als auch offene Fragen und Problemstellungen der Performativen Religionspädagogik erneut kritisch reflektiert werden.

38 Vgl. Roth (Anm. 34), 39-45.

39 Ebd., 45.

40 Vgl. ebd., 45.

41 Erika Fischer-Lichte, Die Entdeckung des Zuschauers. Paradigmenwechsel auf dem Theater des 20. Jahrhunderts, in: dies. (Hg.), Die Entdeckung des Zuschauers. Paradigmenwechsel auf dem Theater des 20. Jahrhunderts, Tübingen/Basel (Francke) 1997, 938,35 .

42 Roth (Anm. 34), 49. 


\section{Performanceorientierte Kunstdidaktik}

Auch in der Kunstdidaktik und -pädagogik wird in den letzten Jahren immer häufiger von „Performance“ oder „performativen Verfahren“ gesprochen. ${ }^{43}$ Hierin spiegelt sich nicht nur die allgemeine Konjunktur des Performativen wider, sondern auch eine in der Kunstdidaktik zu beobachtende Tendenz, didaktische Prozesse an künstlerischen Prozessen und Kunstwerken zu orientieren. Unter Bezeichnungen wie „Künstlerische Bildung“ oder „Künstlerische (Feld-)Forschung“ werden Entwürfe versammelt, die ihren didaktischen Ausgangspunkt in der (zeitgenössischen) Kunst wählen. ${ }^{44}$ In diesem Sinne konzentrieren sich einige kunstdidaktische Ansätze auch auf ein vornehmlich künstlerisches Performanceverständnis, das ich im Folgenden zur präziseren Unterscheidung mit Performance-Art bezeichnen möchte. ${ }^{45}$ Zur Konturierung dieses Begriffs sollen zuerst einige wesentliche Merkmale der Performance-Art zusammengetragen und in diesem Horizont kunstdidaktische Überlegungen vorstellt werden. ${ }^{46}$

43 Vgl. Georg Peez, Einführung in die Kunstpädagogik, Stuttgart (Kohlhammer) 3. vollständig überarbeitete Ausgabe 2008, 95; vgl. ähnlich Ulrich Heimann, Störungen Kunstdidaktische Reflexionen über Flash Mob, Intervention, Performanz, in: Joachim Kettel (Hg.), Künstlerische Bildung nach PISA. Neue Wege zwischen Kunst und Bildung, Oberhausen (Athena) 2004, 202-210.

44 Vgl. hierzu exemplarisch die unterschiedlichen Ansätze, die unter der Sammelbezeichnung „Künstlerische Bildung“ oder „Künstlerische (Feld-)Forschung“ subsumiert werden. Vgl. exemplarisch Carl-Peter Buschkühle (Hg.), Perspektiven künstlerischer Bildung. Texte zum Symposium ,Künstlerische Bildung und die Schule der Zukunft', Köln (Salon) 2003; ders., Die Welt als Spiel. II. Kunstpädagogik. Theorie und Praxis künstlerischer Bildung, Oberhausen (Athena) 2007; Helga Kämpf-Jansen, Ästhetische Forschung. Wege durch Alltag, Kunst und Wissenschaft. Zu einem innovativen Konzept ästhetischer Bildung, Köln (Salon) 2001; Kettel (Anm. 43); Andreas Brenne, Ressource Kunst. ,Künstlerische Feldforschung in der Primarstufe. Qualitative Erforschung eines kunstpädagogischen Modells, Münster (Monsenstein und Vannerdat) 2004.

45 Vgl. ähnlich Marie-Luise Lange, Grenzüberschreitungen. Wege zur Performance, Königstein/Taunus (Helmer) 2002, 21-30; Joachim Fiebach, Art. Performance, in: Ästhetische Grundbegriffe Bd. 4, 2002, 740-758.

46 Vgl. den umfassenden Überblick bei Marie-Luise Lange, Schneisen im Heuhaufen. Formen von Performance-Art, in: Hannelore Seitz (Hg.), Schreiben auf Wasser. Performative Verfahren in Kunst, Wissenschaft und Bildung, Bonn/Essen (Kulturpolitische Gesellschaft) 1999, 149-161; dies., Performance zwischen Aufführungskunst und kommunikativer Praxis, in: Kunst+Unterricht 273, 2003, 22 f. Einen Überblick über die kunstgeschichtliche Entwicklung und aktuelle Tendenzen der Performance-Kunst bieten Fiebach (Anm.45), 741-747; Thomas Dreher, Performance Art nach 1945, München (Fink) 2001; Rose Lee Goldberg, Performance Live Art since the 60 s, London (Thames and Hudson) 1998; Elisabeth Jappe, Performance. Ritual. Prozeß, München/New York (Prestel) 1993; Petra Maria Meyer, Performance im medialen Wandel. Einleitender Problemaufriss, in: dies. (Hg.), Performance im medialen Wandel, München (Prestel) 2006, 35-75. 
Performance-Art kann unter die Aktionskünste gefasst werden, bei denen zentral ist, „daß sich der ästhetische Prozeß durch das körperliche Handeln der Akteure innerhalb eines ,Echt-Zeit-Raumes' (Daniel Charles) entfaltet. " ${ }^{47}$ Die bestimmenden Faktoren der Performance-Art sind somit die Dauer, der Ort und die gestische Aktionspräsenz der Künstlerinnen und Künstler. Neben diesen Grundfaktoren zeichnet sich PerformanceArt durch Intermedialität (synästhetischer Einsatz von Sprache, Geräuschen, Musik, Licht, Alltagsgegenständen usw.) und Interdisziplinarität (Cross-over aus Theater, bildender Kunst, Musik, usw.) aus. Dabei stehen die Akteure in einem unmittelbaren Bezug zum Publikum, ohne dieses jedoch - wie z. B. häufig bei Happenings - in die Performance direkt mit einzubeziehen. ${ }^{48}$ Dennoch ist das Publikum an den Performances aktiv beteiligt. Denn für Zuschauerinnen und Zuschauer sowie Künstlerinnen und Künstler gilt im gelingenden Falle, dass sie beide - je auf ihre Art - im performativen Akt „verändert“, „transformiert“ werden. Dabei sind Performances vor allem „eine Ich-Aktion [...], in der sich dieses ,Ich“ öffentlich ,performiert', gleichsam mit Aktion oder Handlung ,maskiert “ “49 Performancehandeln ist somit eine selbstgenießende Entdeckung und Erfindung des Selbst, ist ein Handeln, in dem etwas von einem verborgenen Ich aufscheint, das über das alltäglich Erlebte hinausweist und neue Perspektiven eröffnet. ${ }^{50}$ In dieser subjektorientierten Zuspitzung des Performancebegriffs werden zwar noch Schnittmengen zur Theaterinszenierung (im Sinne von Fischer-Lichte) sichtbar, deutlich wird aber auch, dass Performance-Art hierin nicht aufgeht. Denn im Unterschied „zum Theater, das auf die ,Welt' verweist - erzählt die Performance von nichts anderem als

47 Lange (Anm. 46), 149 (im Original kursiv). Meyer problematisiert jedoch angesichts der fortschreitenden Mediatisierung von Performances die Betonung des „Präsenz-Charakters“. Denn auch die „Live-Performance erweist sich als immer schon mediatisiert“ (Meyer (Anm. 46), 64) und ist damit nicht ausschließlich an die Präsenz der Künstlerinnen und Künstler sowie die Einmaligkeit und Ereignishaftigkeit der Aktionen gebunden. In diesem Sinne konstatiert an anderer Stelle Lange, dass durch den Einsatz elektronischer Medien die unmittelbare Anwesenheit der Künstlerinnen und Künstler nicht zwingend erforderlich sei. (Vgl. Lange (Anm. 45), 31.)

48 Vgl. Lange (Anm. 46), 150 f; dies. (Anm. 45), 27 ff; 38.

49 Hans-Martin Ritter, Mutmaßungen über die ,Performance' und über den Innenraum einer besonderen Form öffentlicher Äußerung, in: Seitz (Anm. 46), 218-224, 221. Schmitz problematisiert jedoch die Suche nach körperlicher resp. subjektiver Authentizität im performativen Akt (wie auch in der Kunst im Allgemeinen). Er sieht hierin eine historisch bedingte, „romantische“ Suche nach verlorener Ursprünglichkeit und Echtheit. Vgl. Norbert M. Schmitz, Der Diskurs über Performance und der Mythos des Authentischen. Eine Kunstform als Übung zivilisatorischer Alltagsästhetik, in: Meyer (Anm. 46), 441-461.

50 Vgl. Lange, M.-L., „....und setzte mich frei“. Von der Kunst, sich im Handeln zu finden, in: Buschkühle (Anm. 44), 237-247, $245 \mathrm{f}$. 
eben von diesem Ereignis, das sich ereignet. Sie verweist auf nichts als sich selbst. “51

Gerade in dieser konsequenten Ich-Orientierung erblickt die Kunstpädagogik Bildungschancen für das ästhetisch tätige Subjekt. Eingebettet in einen pädagogischen Prozess werden „performative Elemente subjektiver künstlerischer Verausgabung Momente eines gesellschaftlichen Sinngebungsprozesses, der auf etwas zielt, was nicht selten mit einem anscheinend banalen Wort bezeichnet wird: Bildung. “ ${ }^{52}$ Nach Marie-Luise Lange handelt sich hierbei um einen „Balanceakt zwischen körperlich-biografischer Vergegenwärtigung und selbstreferentiellem Spiel mit ästhetischen Mitteln “53. Damit steht eine solche pädagogische Performancearbeit insbesondere im Dienst der Sinn-Suche der Heranwachsenden. ${ }^{54}$ Ziel performativer, prozessorientierter Aktivitäten ist die Ausbildung von sinnlich-körperlichen und intermedial-ästhetischen Vorstellungs- und Ausdruckskräften der lernenden Subjekte sowie die Entwicklung von praktisch-suchenden Handlungsmodellen. ${ }^{55}$

Kunstdidaktische Arbeit orientiert sich in ihrer Auseinandersetzung mit Performance-Art an künstlerischen Strategien der Gegenwartskunst und besitzt im konkreten eigenen performativen Tun einen unmittelbaren Bezug zum ästhetisch tätigen Subjekt, das „poietische Eigenleistungen“56 erbringt. In didaktisch-hermeneutischer Perspektive geschieht dies in der Performancearbeit durch subjektive Raum-, Zeit- und biografisch-facettierte Körpererfahrungen, durch eine Nähe zum „Als-Ob“ des Spiels, durch ein Oszillieren zwischen Zufall und Konzept, Chaos und Ordnung. ${ }^{57}$ Dabei bedarf eine so ausgerichtete Performancearbeit einer intensiven Übung. Um die Lernsubjekte überhaupt zu befähigen, mit sich, ihren Körpern und den anderen Teilnehmenden in Kontakt zu kommen, muss eine angemessen Entblockung stattfinden. ${ }^{58}$ Die Schülerinnen und Schüler müssen lernen, ihren Körper als Werkzeug zu begreifen und diesen in eigenen

51 Ritter (Anm. 49), 221.

52 Ulrike Hanke, Von Wildwüchsen, Maulwürfen und Gärtnern. Performance und Lehre, in: Seitz (Anm. 46), 94-101, 96.

53 Marie-Luise Lange, Die Spur führt immer zu uns zurück, in: Angela Ziesche/Stefanie Marr (Hg.), Rahmen aufs Spiel setzen, Königstein/Taunus (Helmer) 2000, 155.

54 Vgl. Andrea Franke, Aktuelle Konzeptionen der ästhetischen Erziehung, Freiburg (Meidenbauer) 2007, 284.

55 Vgl. Lange (Anm. 45), 311.

56 Lange, M.-L., „...it happens...“ Kreuzfahrten des Performativen, in: Kunst+Unterricht 273, 2003, 4-10, 10.

57 Vgl. Lange (Anm. 45), 310; vgl. a. dies., Über Lebendigkeit oder die Präsenz des (Un-) Sichtbaren, in: dies. (Hg.), Performativität erfahren. Aktionskunst lehren - Aktionskunst lernen, Berlin (Schibri) 2006, 101-142, $102 \mathrm{ff}$.

$58 \mathrm{Vgl}$. Franke (Anm. 54), 289. 
Kommunikationsräumen anzuwenden. ${ }^{59}$ In performanceinitiierenden Kursen haben sich dabei drei aufeinander aufbauende Phasen bewährt: ${ }^{60}$ eine Lockerungs- und Konzentrationsphase, eine Experimentier- und Erprobungsphase sowie eine eigenständige Erprobungsphase bzw. eine Konzeptions- und Aufführungsphase der eigenen Performance. Dabei dienen elementare Themenstellungen, wie z.B. Nackt- und Verhüllt-Sein, Körper, Krieg oder Streit als lockere Bezugsrahmen ${ }^{61}$ um die Performancearbeit zu initiieren. Die Themen stehen daher im Dienste (subjektorientierter) künstlerischer Prozesse. Eine so strukturierte Performancearbeit funktioniert nach Lange in der Spannung zwischen dialogischen Gruppen- und Ich-bewussten Einzelaktionen, die sowohl Körperarbeit als auch Vertrauensbildung umfassen und sich spielerisch zwischen Improvisation und Konzept, Regel und Regelüberschreitung erstrecken sollten. ${ }^{62}$

\section{Performative Religionspädagogik im Horizont von kunstdidaktischer Performancearbeit}

Performative Dimensionen sind - um die Unterscheidung von Fischer-Lichte noch einmal aufzugreifen - in der performativen Religionspädagogik unterschiedlich deutlich ausgeprägt. In den dargestellten kunstdidaktischen Ansätzen tritt nun diese performative Dimension explizit in den Vordergrund. Die Performancearbeit in der Kunstdidaktik lässt somit erahnen, wie eine in diesem Sinne entschieden performativ ausgerichtete Religionsdidaktik aussähe. Zum einen müsste unterrichtspraktisch nach den Möglichkeiten performanceinitiierenden Arbeitens im RU gefragt werden, denn die geschilderten Phasen der Performancearbeit erfordern nicht nur viel Zeit, sondern auch hohe Kompetenzen von Lehrerinnen und Lehrern sowie Schülerinnen und Schülern. Zum anderen gilt es die strikte Subjektorientierung performanceorientierter Kunstdidaktik religionsdidaktisch zu reflektieren. Die (Referenz-)Themen bilden zumeist ausschließlich den Rahmen oder Ausgangspunkt der (Lern-)Prozesse, deren Inhalt das agierende Subjekt mit seiner körperlich-biografischen Vergegenwärtigung selbst ist, sie dient primär der Selbstbildung der lernenden Subjekte. Nun steht re-

59 Vgl. Johannes BBB Deimling, Eigene Leistung. Der Rechtschreibfehler ist das, Ähm` der Unterhaltung, in: Lange (Anm. 57), 50-76, 57.

60 Vgl. Lange (Anm. 45), 402 ff; vgl. ähnlich Andrea Wuttke, Spannungsfeld Körper, Raum, Zeit. Einblicke in die ,Aktionswerkstatt' einer 6. Klasse, in: Kunst+Unterricht 273, 2003, 31-34. Ähnliche Strukturen entdeckt man bei der Analyse dokumentierter Praxisbeispiele. Vgl. Deimling (Anm. 59); Petra Maria Peters, Performative Handlungen und biographische Spuren in Kunst und Pädagogik, Hamburg (Uni-Press) 2005.

61 Vgl. z. B. Wolfgang Sautermeister, Jetzt. Notizen zur Lehrbarkeit von Performance Kunst, in: Lange (Anm. 57), 77-88.

62 Vgl. Lange (Anm. 57), 107. 
ligionspädagogisch die Bedeutung von Selbstbildung und Subjektorientierung außer Frage. Wenn es der Performativen Religionspädagogik jedoch (auch) darum geht, die konkrete Religion darzustellen, sie „in's Spiel“ zu bringen, dann stellt sich die Frage, wie diese Formen (und m. E. wäre zu ergänzen: die Inhalte) in der Performance „in’s Spiel“ gebracht werden können. Wenn mit Marie-Luise Lange pädagogische Performancearbeit ein „Balanceakt zwischen körperlich-biografischer Vergegenwärtigung und selbstreferentiellem Spiel mit ästhetischen Mitteln "63 ist und vor allem der individuellen Sinn- und Identitätssuche dient, sind dann die religiösen Formen die ästhetischen Mittel im selbstreferentiellen Spiel der Performance? Oder tragen sie zu Erfahrungen körperlich-biografischer Vergegenwärtigung bei, die für religiöse Lernprozesse relevant sind? Welche religiösen Formen, Texten oder Themen können mit einer solchen Subjektorientierung einhergehen? Einzelne Gebete oder Gebetshaltungen bieten sicherlich mehr Anknüpfungspunkte für einen so ausgerichteten performativen RU als z. B. die Eucharistiefeier. Denn am Beispiel der Eucharistie dürfte deutlich werden, dass dieser zwar eine performative Grundstruktur eigen ist, die nur im Vollzug darstellbar ist. Aber es ist fraglich, ob hieraus auch didaktische Prinzipien abzuleiten sind. Es ist nur schwer vorstellbar, dass sich Schülerinnen und Schüler Eucharistie in didaktischen Kontexten performativ ansatzweise erschließt, wenn der religiöse Kontext fehlt.

Gerade wenn die performative Religionspädagogik von einem radikalen Traditionsabbruch ausgeht, so dass Religion zuerst gezeigt und zumindest ansatzweise angeeignet werden muss, bevor sie im RU reflektiert werden kann, dann stellt sich die Frage, was in und durch solche (subjektorientierte) Performancearbeit erfahren wird. Kann angesichts von Traditionsabbruch Religion im schulischen Kontext performativ angeeignet oder transformiert werden, oder braucht es hierzu nicht doch - im Sinne Austins ernsthafte und aufrichtige Vollzüge innerhalb einer religiösen Gemeinschaft $?^{64}$ Und noch komplizierter: Was passiert, wenn einzelne Schülerinnen und Schüler die Performance eher spielerisch experimentell, andere wiederum als ernsthaften und aufrichtigen religiösen Vollzug wahrnehmen?

Mir scheint, dass hierbei die stets betonte Notwendigkeit der unterrichtlichen Reflexion performativen Handelns nicht weit genug reicht - und je nach kognitiven und entwicklungspsychologischen Voraussetzungen der Schülerinnen und Schüler auch nicht so weit reichen kann. ${ }^{65}$ Reflexion und performatives Handeln bleiben vielfach zwei getrennte Einheiten mit unterschiedlicher Erfahrungsintensität. Es ist m.E. sinnvoller, Dimensio-

63 Lange (Anm. 53), 155.

64 Nach Lämmermann braucht Performatives ein „begrenztes Umfeld und ein geteiltes Vorverständnis, sonst funktioniert es ganz einfach nicht.“ (Lämmermann (Anm. 1), 113.)

65 In dieser Hinsicht problematisiert Roose insbesondere performatives Lernen in der Grundschule. Vgl. Roose (Anm. 33), 108 f. 
nen von Reflexion, von Wissens- und Kenntniserwerb in das performative Handeln zu integrieren. Erinnert sei in diesem Zusammenhang an Ursula Roths aus der Theaterwissenschaft und -didaktik abgeleiteter Vorschlag, didaktische Inszenierungen in Brechtscher Manier zu verfremden, zu durchbrechen. Hierdurch ist der (performativen) Darstellung bereits ein reflexives Moment inhärent.

Hingewiesen werden kann analog auch auf kunstdidaktische Überlegungen, die das eigene gestalterische Tun als einen umfassenden Prozess strukturieren, in dem Kunstrezeption und eigenes Gestalten, Recherche und Wissenserwerb ebenso einfließen wie die persönliche, ästhetische Auseinandersetzung mit vorgegebenen Themen (Künstlerische Bildung; Künstlerische (Feld-)Forschung). ${ }^{66}$ Analog wäre ein RU zu denken, in dem performative Elemente in umfassenden religionsdidaktisch entwickelten Projekten $\mathrm{zu}$ verorten wären. Damit würde jedoch das, was gegenwärtig auf der Ebene einer „performativen Religionspädagogik“ bzw. „Religionsdidaktik" diskutiert wird, auf eine didaktisch-methodische Ebene verschoben. ${ }^{67}$

\section{Zusammenfassung}

Die so genannte „Performative Religionspädagogik“ ist gegenwärtig ein Sammelbecken vielfältiger Ansätze. Insbesondere der Begriff „performativ“ wird dabei zu einem „umbrella term“, unter dem höchst Unterschiedliches versammelt wird. Das Bedeutungsspektrum dieses Begriffs reicht von John L. Austins performativen Sprechakten bis hin zu einem theater- oder kulturwissenschaftlich geprägten Performanceverständnis. Dieser äquivoke Begriffsgebrauch führt so zu höchst unterschiedlichen didaktisch-hermeneutischen Ausprägungen und zu teils vehementen Diskussionen über die Stärke und Reichweite der Performativen Religionspädagogik. Ein Blick in die (kunst-)didaktisch bereits etablierte Performancearbeit kann in dieser Hinsicht sowohl zu einer Begriffsschärfung als auch zu einer stärkeren didaktisch-hermeneutischen Konturierung der Performativen Religionspädagogik beitragen.

\section{Abstract}

The so-called "Performative Religionspädagogik" presently serves as a gathering point for various approaches. Particularly, the term "performative" is turning into an umbrella term sheltering highly different projects. Its meaning varies from John Austin's "performative utterances" to the concept of "performance" in cultural studies. The heterogeneous

66 Vgl. Anm. 44.

67 Ähnlich argumentiert Rothgangel, für den „das Potential des performativen Ansatzes primär in ,religionsmethodischer' Hinsicht liegt“". (Vgl. Martin Rothgangel, [Rezension zu:] Thomas Klie/Silke Leonhard (Hg.), Schauplatz Religion. Grundzüge einer Performativen Religionspädagogik, Leipzig 2003, in: Theologische Literaturzeitung 129, 2004, $577-579,579$. 
application leads to different didactic-hermeneutic consequences and, thus, vigorous discussions of the strength and weaknesses as well as the scope of the "Performative Religionspädagogik." This article focuses on the didactics of art and the well-established performance art, hence, seeking to contribute to a sharper comprehension and a more distinct didactic-hermeneutic conception of the "Performative Religionspädagogik." 